\title{
INDICADORES DE CALIDAD FÍSICA EN SUELOS DEL CHACO SEMIÁRIDO BAJO DISTINTOS SISTEMAS
}

\section{Physicals indicators of soil quality in the Semi-Arid Chaco Area under different systems}

\author{
Urinovsky Irigoyen, Kevin M.; Toledo, Diana M.; Arzuaga, Silvia A.; Acosta, María G. L.; Contreras \\ Leiva, Stella M. ${ }^{1}$ \\ ${ }^{1}$ Cátedra de Edafología - Facultad de Ciencias Agrarias -Universidad Nacional del Nordeste. \\ Sargento J.B. Cabral 2131. Corrientes (3400). e-mail: marcelatoledo94@hotmail.com
}

\begin{abstract}
RESUMEN
El objetivo del presente trabajo fue evaluar en Molisoles del Chaco semiárido, el impacto del uso del suelo sobre algunos atributos físicos de calidad. Se muestrearon 3 sistemas: un sistema de monte nativo como referencia de alta calidad de suelos (Mon), un sistema silvopastoril de, monte nativo, con una pastura implantada (Sil) y un sistema agrícola bajo siembra directa (SD) con soja como cultivo principal. Se determinaron las siguientes variables: $\mathrm{pH}$, densidad aparente (Da), humedad equivalente (HE), conductividad eléctrica (CE), textura y estabilidad de agregados (EA). Los suelos bajo estudio resultaron de reacción neutra y de textura franco-arcillosa a franco-arcillo-arenosa. El desmonte y posterior uso agrícola bajo SD, provocó cambios desfavorables como densificación, disminución de la capacidad del suelo para retener agua para los cultivos y disminución de la estabilidad de los agregados, impactando negativamente sobre la calidad física. El sistema silvopastoril produjo un impacto menor, ya que, si bien produjo densificación, y disminuyó la capacidad de retención de agua, el efecto sobre los agregados y su estabilidad fue menor, tomando valores medios entre 85 y $80 \%$, cercanos al Mon $(85 \%)$, y diferenciándose estadísticamente del sistema agrícola. La EA resultó un buen indicador de calidad, de relativa facilidad y bajo costo de determinación. El orden decreciente de calidad física del suelo en base al índice de estabilidad de agregados fue Mon $\geq$ Sil $>$ SD
\end{abstract}

Palabras clave: calidad de suelo, desmonte y cambio de uso, sistema silvopastoril, sistema agrícola bajo siembra directa

\begin{abstract}
The aim of this work was to assess the impact of soil use on some physical quality attributes on Molisoles soils from semi-arid Chaco in Argentina. Three soils use systems were sampled: i) a native forest system taken as high soil quality reference (Mon), ii) a silvopastoral system with pastures (Sil), and iii) an agricultural no-till system (SD) having soy as the main crop. The following variables were determined: $\mathrm{pH}$, bulk density (Da), equivalent humidity (HE), electrical conductivity (CE), texture, and aggregate stability (EA). These soils proved to have a neutral reaction, and a texture ranging from clay loam to sandy clay loam. Forest clearing and the subsequent agricultural use under no-till caused unfavorable changes such as densification, a decrease in soil capacity to retain water for crops, and aggregate stability which resulted in an adverse effect on soil physical quality. The silvopastoral system produced a smaller impact, even though it enhanced densification and decreased water retention capacity, the effect on aggregates and their stability was lower with average values between 85 and $80 \%$, close to native forest values $(85 \%)$, and statistically different from the agricultural system. Aggregate stability proved to be an easy-to-use inexpensive reliable indicator of soil quality. The decreasing order of physical soil quality based on aggregate stability index was Mon $\geq$ Sil $>$ SD.
\end{abstract}

Key words: soil quality, forest clearing and land-use changes, silvopastoral system, the no-till agricultural system.

Recibido: 6/Jul/2020. Aceptado: 19/Feb/2021 
Urinovsky Irigoyen, K.M.; et al. (2021) Indicadores de calidad física en suelos en suelos del Chaco semiárido...

\section{INTRODUCCIÓN}

La ampliación de la frontera agropecuaria es un proceso que se viene desarrollando en la Argentina a expensas del territorio cubierto por vegetación natural como pastizales y montes nativos (Rosso, 2014). Vigglizzo y Jobbágy (2010), estudiaron la dinámica de la frontera agropecuaria y el cambio tecnológico en la Argentina, estimaron que la expansión territorial de los cultivos de secano ocurrió a expensas de las tierras de bosques $(-18,4 \%)$ y de pastizales $(-6,8 \%)$, y destacaron un gran incremento del área asignada a cultivos anuales en el Chaco Subhúmedo Occidental. Gran parte del aumento de la producción de alimentos se ha llevado a costa de centenares de millones de hectáreas de bosques, si bien no hay estimaciones sólidas sobre la superficie de tierras agrícolas y de pastoreo que originalmente estaban cubiertas de bosques, lo cierto es que una gran proporción de éstos fue talada para dar lugar a actividades agrícola-ganaderas (Rosso, 2014). Si bien este proceso de desmonte se manifiesta en gran parte en el centro y norte del país, es el Bosque Chaqueño en donde actualmente se ubica la mayor parte de esas áreas con desmonte activo, principalmente en las provincias de Salta, Tucumán, Chaco, Santiago del Estero y Córdoba (Montenegro et al., 2004). El cambio en el uso de las tierras, los sistemas de cultivo y las prácticas de manejo, producen cambios en las propiedades del suelo y cuando no se realizan prácticas tendientes a protegerlo y conservarlo, el uso conlleva a una pérdida de calidad del suelo, a una degradación que puede ser física, química y/o biológica (Porta Casanella et al., 1999).

La definición de calidad de suelo (CS), es la capacidad del suelo para funcionar dentro de ciertos límites naturales y antrópicos del ecosistema, sustentar la productividad vegetal y animal, mantener la calidad del agua y del aire, promover la salud de plantas, de animales y del hombre, y soportar su habitabilidad (Doran \& Parkin, 1994).

La salud del suelo se ha definido como su capacidad para funcionar como un sistema vivo. Los suelos sanos mantienen una comunidad variada de organismos del suelo que ayudan a controlar las enfermedades de las plantas, insectos y malezas, forman asociaciones simbióticas beneficiosas con las raíces, reciclan nutrientes esenciales para las plantas, mejoran la estructura del suelo con efectos positivos para el agua del suelo y la capacidad de retención de nutrientes, y en última instancia mejoran la producción agrícola. (FAO, 2015). Es decir que la CS, no sólo está relacionada con su productividad efectiva, sino también con la calidad ambiental y la salud de las plantas, de los animales y del hombre, con la vida del suelo. Como concepto integrador la CS no puede ser medida directamente, pero si cuantificada a través de un grupo indicadores que por su sensibilidad y facilidad de medición, permiten comparar distintos usos y manejos (Brejda et al., 2000).

Dalurzo (2002), Rojas (2012), Toledo (2014), señalan como indicadores de calidad para suelos del nordeste argentino, a los atributos físicos: densidad aparente, humedad equivalente, estabilidad de agregados, densidad de partículas, resistencia a la penetración, infiltración, porosidad total, porosidad capilar, estabilidad estructural en seco, espacio aéreo, fracción erosionable. Estos atributos pueden sintetizarse en el marco de la evaluación de la estructura, la cual es dinámica y el resultado de muchos factores y procesos abióticos y bióticos. Los principales condicionantes de la formación y estabilización de la estructura son la textura, la materia orgánica, los organismos del suelo, la profundidad de la napa de agua y las condiciones climáticas (Kooistra \& Tovey, 1991), asociado a procesos de expansión y contracción (durante el humedecimiento y secado) que se incrementan con el contenido de arcilla (Dexter, 1988).

Los agregados del suelo son las unidades básicas de la estructura y son el resultado del reacomodamiento de las partículas la floculación, cementación y del mismo. Estas unidades estructurales definen las propiedades físicas y mecánicas del suelo, tales como retención de agua, movimiento del agua, aireación y temperatura, que a su vez afectan los procesos físicos, químicos y biológicos. La formación de agregados involucra numerosos factores: vegetación, fauna del suelo, microorganismos, cationes e interacciones entre partículas de arcilla y materia orgánica (Kumar et al., 2013 citado por Costa et al. 2018). La estabilidad de los agregados depende de su cohesión interna, volumen de poro, conectividad, tortuosidad e hidrofobicidad de la pared de poro (Chenu y Cosentino, 2011).

La estabilidad de los agregados es utilizada frecuentemente como un indicador de la estructura del suelo, y es una medida de la capacidad de las unidades estructurales del suelo para mantener la arquitectura de la fracción sólida y del espacio poroso bajo la acción del agua o la aplicación de un estrés mecánico externo (Dexter, 1988). 
En la mayoría de los suelos la materia orgánica (MOS) es el principal agente que contribuye y estimula la formación y estabilización de agregados suelo (Golchin et al., 1994), como asi también el contenido de arcilla y los efectos de la contracción y expansión en suelos ricos en arcillas 2:1. Oades (1984) propuso un modelo de formación de agregados en el cual los microagregados serían inicialmente formados dentro de los macroagregados del suelo, la cual fue confirmada por Six et al. (2000). Cabe destacar, que para el caso de suelos Molisoles y Vertisoles, los principales mecanismos de agregación son el contenido y tipo de arcillas respectivamente (Utomo y Dexter, 1982), y. la materia orgánica del suelo (Oades, 1993).

De manera que el estudio de la estabilidad estructural, es importante para entender el efecto de los factores externos sobre el suelo, en particular los de origen antrópico y para prever las modificaciones de su funcionamiento y el efecto resultante sobre la calidad física del suelo. La EA hace referencia a la habilidad de los agregados del suelo a resistir la disrupción cuando son aplicadas fuerzas externas (usualmente asociadas al agua) (USDA, 1996).

La EA es un indicador temprano de reconversión o de degradación del suelo y es un indicador del contenido de la calidad física, de la materia orgánica, de la actividad biológica y del ciclado de nutrientes en el suelo USDA (1996). En general, las partículas están unidas formando pequeños agregados $(<0,25 \mathrm{~mm})$ por medio de formas más antiguas y más estables de materia orgánica. La descomposición microbiana de la materia orgánica fresca libera productos (que son menos estables) que unen agregados pequeños en agregados grandes $(>2-5$ $\mathrm{mm}$ ). Estos grandes agregados son más sensibles a los efectos del manejo de la materia orgánica, y sirven como un mejor indicador de los cambios en la calidad del suelo. Mayores cantidades de agregados estables sugieren una mejor calidad del suelo, Cuando aumenta la proporción de agregados grandes a pequeños, la calidad del suelo generalmente aumenta, el tamaño, distribución y forma de los poros creados favorecen el desarrollo de las raíces y estructura (Six et al., 2004). El porcentaje de agregados estables, está dado por aquellos que resisten la acción del agua sin romperse ante su fuerza disturbadora e indica la proporción de agregados que resiste a la perturbación, de manera que una mayor cantidad de agregados estables es un indicador de una mayor estabilidad estructural. Una buena EA favorece la permanencia de una buena relación aire-agua para el desarrollo de las plantas y la biota del suelo, contribuyendo a una mejor calidad física del mismo (USDA, 2011).

Muchos estudios han demostrado que la producción de EPS (sustancias poliméricas extracelulares) puede aumentar la agregación del suelo, mejorar la calidad del suelo y contribuir a la fertilidad del suelo, ya que además de mejorar la estructura del suelo, puede mejorar la absorción de nutrientes y la disponibilidad de agua tanto para las plantas como para los microorganismos, beneficiando al medio ambiente en su conjunto (Costa et al., 2018).

Las prácticas de manejo, tales como la labranza, la rotación de cultivos, la aplicación de fertilizantes, y el manejo de los residuos, también influyen en las transformaciones de la MOS, en las propiedades físicas del suelo (Gregorich et al., 1994; Ladd et al., 1994; Haynes, 2000), en la actividad de los organismos, en los pegamentos producidos por hongos y bacterias y por consiguiente en la estructura y en la estabilidad de los agregados (González-Chávez et al., 2004).

Angers y Mehuys (1988) señalaron que los cultivos ejercen un gran efecto sobre los agregados estables en agua correspondientes a la fracción de 2-6 mm. y que la agricultura continua con labranza convencional, en Argiudoles típicos, disminuye el nivel de agregación y la resistencia al colapso por humedecimiento.

En particular, en la Provincia del Chaco la agricultura, la ganadería y la explotación forestal, han modificado profundamente la composición de las comunidades vegetales, sea destruyendo el estrato herbáceo y facilitando la invasión por especies arbustivas, sea eliminando las especies forestales más valiosas, o bien talando o quemando totalmente el bosque para crear campos destinados a la agricultura (Rojas, 2012), impactando no solo en el ambiente sino también en los suelos, llegando a producir degradación. Los campos degradados han cambiado su fisonomía original de bosque con pastizales de gran riqueza florística, por un matorral cerrado, las técnicas de recuperación se basan en desmontes selectivos y clausuras para la restauración del bosque y de descansos estratégicos para mejorar la productividad de los pastizales. Los ecosistemas del Chaco árido y semiárido son ambientes con vocación forestal donde la principal actividad es la ganadería y la explotación del bosque se realiza como alternativa de apoyo conformando Sistemas Silvopastoriles. Se utilizan pasturas implantadas y 
entre las especies con mejores cualidades y adaptación se pueden mencionar: Grama Rhodes (Chloris gayana), Gatton panic (Panicum maximun cv. Gatton), Green panic (Panicum maximun var. trichoglume) y Coloratum (Panicum coloratum) (Rossi, 2000).

Venialgo et al., (2004), evaluaron algunas propiedades físicas de los suelos de la ecorregión del Chaco Seco, bajo producción agrícola y distintos tipos de labranza comparándolos con el suelo de monte y concluyeron que el cambio de cobertura y las labranzas producen una disminución de la EA en relación al monte, siendo la siembra directa el sistema que menos afecta la estabilidad. Similares resultados fueron hallados por Gutiérrez et al. (2002) en suelos del Sudoeste del Chaco pertenecientes a la Serie Tizón (Haplustol óxico) bajo cultivo agrícola, donde la estabilidad de agregados y los contenidos de materia orgánica mejoraron en los espesores superficiales y sub-superficiales al incorporar un sistema de labranza conservacionista como la siembra directa. En suelos donde las partículas están agregadas, el diseño y la perdurabilidad del espacio poroso dependen en gran medida de la forma, el tamaño, la distribución y la estabilidad de los agregados, por lo que el movimiento y la disponibilidad del agua, y el aire están influenciados por el estado de agregación (Cabria et al., 2002).

El objetivo de este trabajo fue evaluar en Ustoles del Chaco semiárido, el impacto de la transformación de bosques nativos a sistemas silvopastoriles y de la eliminación del monte nativo y el posterior uso agrícola sobre algunos atributos físicos de calidad.

\section{MATERIALES Y MÉTODOS}

El estudio se llevó a cabo en la Ecorregión Chaco Seco, Subregión Chaco Semiárido y en particular dentro del Complejo Chaco Subhúmedo Central (Morello et al., 2009), entre las isohietas de 800 a $900 \mathrm{~mm}$ (Figura 1).

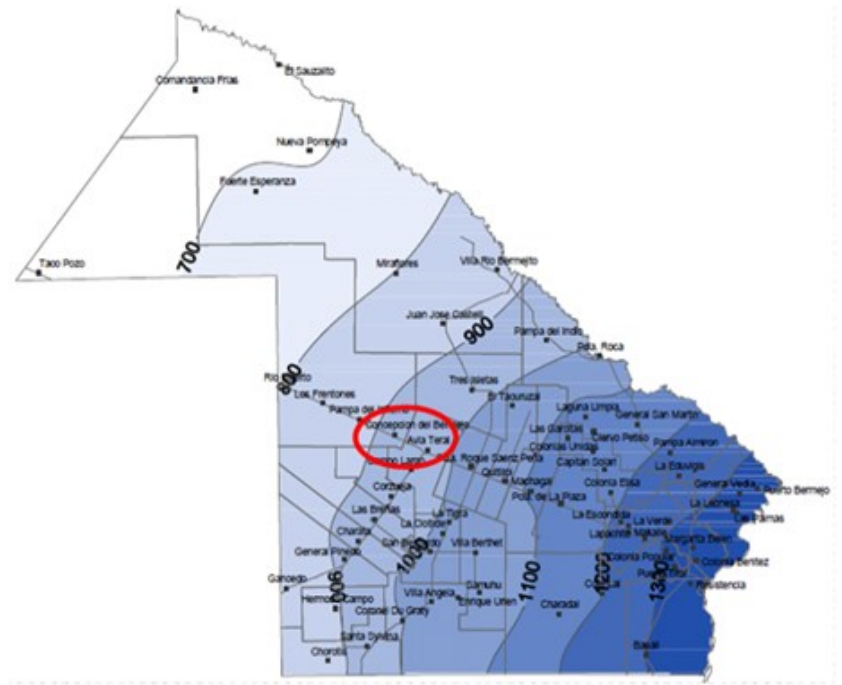

Figura 1. Mapa de Isohietas Medias Anuales de la Provincia del Chaco (1956-2010-Fuente: APA). Con círculo rojo se señala la ubicación del Área de Estudio.

Dentro del Complejo Chaco Subhúmedo Central el clima es mesotermal subhúmedo seco, presentando un déficit hídrico promedio de $200 \mathrm{~mm}$. La precipitación media anual oscila entre 650 a 900 $\mathrm{mm}$. La temperatura media anual es aproximadamente de $21^{\circ} \mathrm{C}$.

En esta investigación se empleó un diseño de muestreo al azar, con tres tratamientos. Se seleccionaron sitios: utilizando imágenes satelitales y mapas de suelos, trabajo en gabinete y salidas a campo, a fin de ubicar suelos del orden de los Molisoles (Ustoles) en establecimientos con cultivo principal de soja bajo siembra directa con rotaciones, con reservas de bosques nativos sin disturbio antrópico y con uso foresto-ganadero en sistemas silvopastoriles con pastura implantada. Se seleccionaron 9 lotes por tratamiento y en cada uno se tomaron muestras compuestas por 3 submuestras en parcelas de $100 \mathrm{~m}^{2}$ (áreas homogéneas y representativas de los lotes para reducir la variabilidad espacial). El total de lotes muestreados fue de 27 (9 lotes x 3 tratamientos). 


\section{Descripción de los tratamientos:}

Sistema natural, Bosque nativo (Mon): Bosques sin disturbio antrópico, semidensos, con dosel de 10 a 15 $\mathrm{m}$ de altura, fueron tomados como referencia de alta calidad de suelo, respecto del cual se compararon los efectos del uso del suelo. Figura 2.

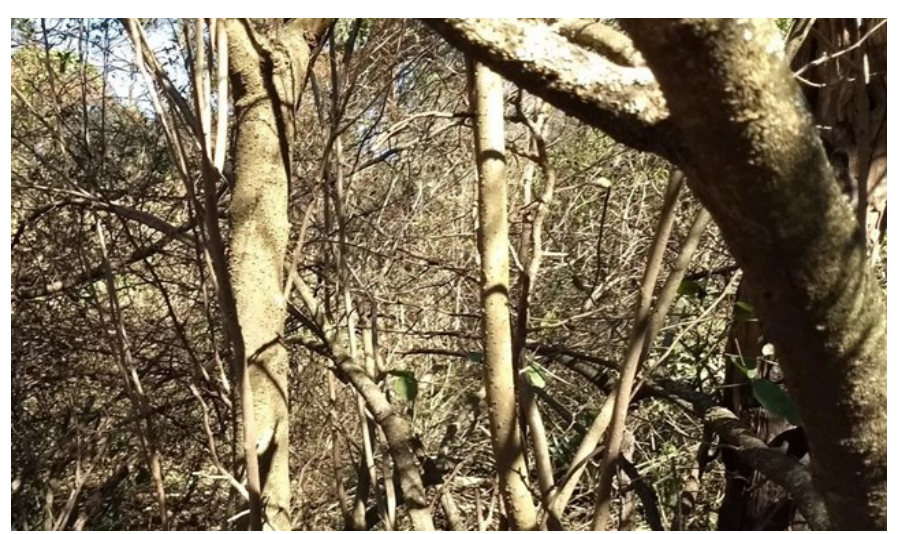

Figura 2. Sistema natural, Bosque nativo

Sistema Silvopastoril con rolado de baja intensidad (SIL): con más de 8 años de habilitación a partir de un bosque nativo con dos pasadas de rolo de baja intensidad con un peso de $3000 \mathrm{~kg}$ a $45^{\circ}$ con siembra simultánea de aproximadamente $5 \mathrm{~kg} \mathrm{ha}^{-1}$ de Panicum máximum cv. Gatton panic y un pastoreo controlado por año (E.V. 1,1 ha $\left.{ }^{-1}\right)$. Figura 3.

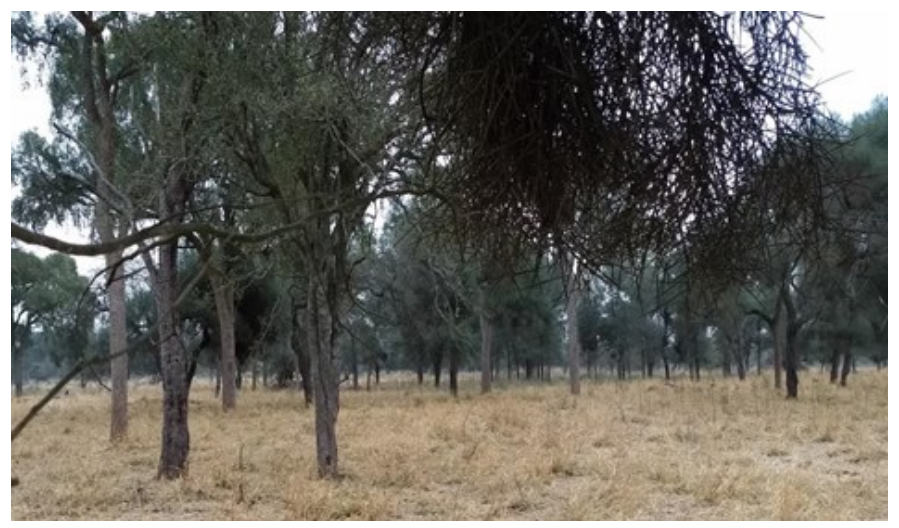

Figura 3. Sistema Silvopastoril con rolado de baja intensidad.

Sistema agrícola bajo siembra directa con rotaciones (SD): lotes desmontados con más de 5 años de agricultura bajo siembra directa, con cultivos anuales en rotación (Soja/Maíz o Soja/Algodón) cuyo cultivo principal es la soja. Figura 4.

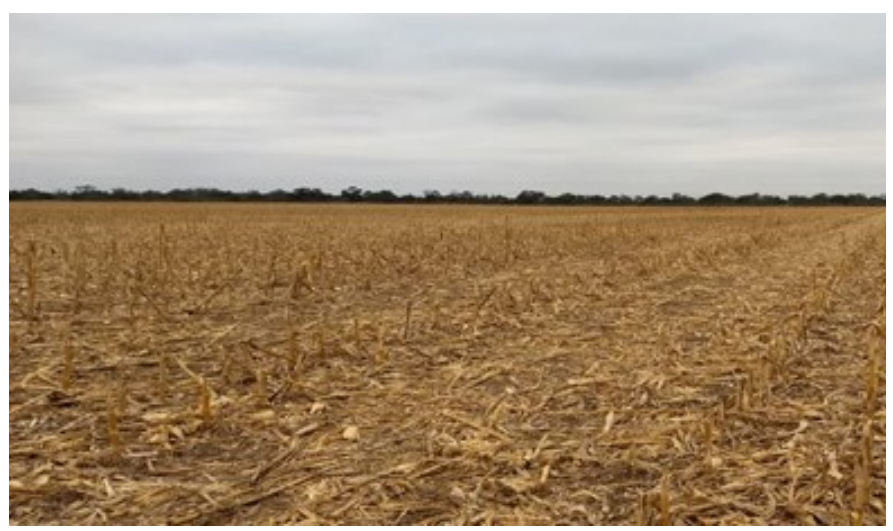

Figura 4: Sistema agrícola bajo siembra directa con rotaciones 
El muestreo de suelo, se realizó a finales de otoño, se efectuó simultáneamente en todos los tratamientos y lotes tomando muestras compuestas en cada lote a dos profundidades: $0-0,05 ; 0,05-0,10$. Los puntos de muestreo fueron georeferenciados.

Las variables determinadas fueron: $\mathrm{pH}$ en agua relación 1:2,5 y conductividad eléctrica (CE) en extracto de pasta saturada (Dewis y Freitas, 1970), textura por el método hidrométrico de Bouyoucos (Dewis y Freitas, 1970), densidad aparente (Da) por el método del cilindro (Forsythe, 1975), humedad equivalente (HE) por el método de la Centrífuga (Montenegro González et al., 1990) y estabilidad de agregados (EA) por el método de (Kemper y Rosenau, 1986).

Los resultados obtenidos fueron analizados mediante análisis de la variancia (ANOVA) y prueba de LSD $(\mathrm{p}<0,05)$ utilizando el software estadístico Infostat Profesional (Di Rienzo et al. 2017).

\section{RESULTADOS Y DISCUSIÓN}

La textura de los suelos varió de franco-arcillosa a franco-arcillo-arenosa, con porcentajes medios de arena entre 38 y 57; de limo entre 22 y 57 y de arcilla entre 20 y 28. El pH presentó valores medios entre 7 y 7,38, siendo la reacción de los suelos neutra, en base al rango propuesto por la USDA (1999).

La CE varió entre 0,32 a $0,76 \mathrm{dS} \mathrm{m}^{-1}$, presentándose los menores valores bajo SD y los mayores bajo SIL, pero en todos los casos permaneciendo entre $0-0,8 \mathrm{dS} \mathrm{m}^{-1}$ considerados aceptables para el crecimiento de los cultivos (Luters y Salazar, 1999) En superficie los sistemas cultivados no se diferenciaron estadísticamente del sistema de referencia (Tabla 1). Similar situación fue encontrada por Rojas et al., (2015), quienes informaron que en suelos del Chaco que las diferencias encontradas en la CE de suelos bajo agricultura y suelos de monte nativo, no fueron significativas.

Las Da de los suelos bajo SD y SIL fueron mayores respecto al Mon para las dos profundidades estudiadas, presentándose en la segunda profundidad el orden SD $>$ SIL $>$ MON, con diferencias significativas $(p<0,0001)$ (Tabla 1). El efecto de la siembra directa sobre la compactación subsuperficial, luego de varios años sin remoción del suelo, es importante, ya que no sólo impacta en la densidad aparente, del suelo sino también en la retención de agua útil para las plantas (Rollán et al., 2004). Al no haber remoción del suelo, el tránsito de maquinaria y el número de pasadas inciden sobre la compactación (Moro et al., 2004). Ferreras et al. (2007), encontraron que los sitios considerados como referencia (situación de alta calidad de suelo) presentaron mejores condiciones de agregación, mayor proporción de carbono orgánico total y menor compactación con respecto al mismo suelo bajo agricultura (rotaciones Maíz-Trigo/Soja y Maíz-Soja-Trigo/Soja con diferentes combinaciones de aplicación de fertilizantes y un Testigo sin fertilizar y ganadería). Por otra parte, en suelos del Chaco, Moro et al. (2004), encontraron en Molisoles del Chaco que sistemas agrícolas con rotaciones bajo labranza cero producen un aumento de la densidad aparente y de la resistencia mecánica a la penetración, respecto de la labranza convencional en el espesor superficial.

Los altos valores de Da bajo SIL, muestran una densificación del suelo, asociada al pisoteo de los animales. Estudios llevados a cabo por Martino (2017), a partir de datos de área basal y peso corporal, reportaron que los animales en pastoreo aplican presiones sobre el suelo en el rango entre 150 (novillo de $300 \mathrm{~kg}$ ) y $350 \mathrm{kPa}$ (oveja adulta), valores notoriamente mayores que los correspondientes a tractores agrícolas, que ejercen presiones del orden de 80 (cubiertas de alta flotación) a $160 \mathrm{kPa}$ (cubiertas radiales simples).

La humedad equivalente (HE) es un parámetro que ha sido utilizado como indicador de calidad de suelos en algunos trabajos y puede revelar diferencias en la capacidad de almacenamiento de agua que tienen influencia en otras propiedades del suelo (Rojas, 2012). El uso del suelo afectó la capacidad del mismo para retener agua para las plantas, con disminuciones de hasta $20 \%$ y 25\% respecto a la situación original de monte para la primera y segunda profundidad respectivamente, con diferencias significativas (Tabla 1). La densificación observada en los sistemas disturbados por el hombre, afecta a la porosidad total del suelo, a la distribución del tamaño de poros y manifiesta su efecto en una disminución apreciable del contenido de agua disponible para los cultivos (Rollán et al., 2004). Los distintos manejos de suelos alteran la velocidad de infiltración de agua y con ello la captación de agua en el perfil, también los cambios en la geometría del espacio poroso inducidos por las labranzas disminuyen la infiltración y la capacidad de retención de agua (Pla Sentís y Ovalles, 1994). El desmonte y posterior uso agrícola afectó la estabilidad de los agregados del suelo (Tabla 1). 
Tabla 1. Valores medios de estabilidad de agregados (EA), densidad aparente (Da), humedad equivalente (HE), conductividad eléctrica (CE), $\mathrm{pH}$ y textura para los sistemas: siembra directa (SD), monte nativo (Mon) y silvopastoril (SIL), a las profundidades de: $0-0,05$ y $0,05-0,10 \mathrm{~m} . \mathrm{n}=9$.

\begin{tabular}{|c|c|c|c|c|c|c|}
\hline Variables & $\begin{array}{l}\text { Prof. } \\
\text { (m) }\end{array}$ & Mon & SIL & $S D$ & $\begin{array}{l}\text { Coeficiente de } \\
\text { variación }\end{array}$ & $P$-valor \\
\hline \multirow[t]{2}{*}{ pH } & $0-0,05$ & $7,10 \mathrm{a}$ & $7,08 \mathrm{a}$ & $7,01 \mathrm{a}$ & 6,70 & 0,9201 \\
\hline & $0,05-0,10$ & $7,15 \mathrm{a}$ & $7,38 \mathrm{a}$ & $7,16 \mathrm{a}$ & 6,11 & 0,4782 \\
\hline \multirow{2}{*}{$\begin{array}{c}\text { Da } \\
\left(\operatorname{Tn~m-~}^{3}\right)\end{array}$} & $0-0,05$ & $1,03 \mathrm{a}$ & $1,16 \mathrm{~b}$ & $1,24 \mathrm{~b}$ & 8,35 & 0,0004 \\
\hline & $0,05-0,10$ & $1,05 \mathrm{a}$ & $1,25 \mathrm{~b}$ & $1,33 \mathrm{c}$ & 5,85 & $<0,0001$ \\
\hline \multirow{2}{*}{ 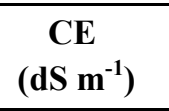 } & $0-0,05$ & $0,66 \mathrm{ab}$ & $0,76 \mathrm{~b}$ & $0,39 \mathrm{a}$ & 57,27 & 0,0888 \\
\hline & $0,05-0,10$ & $0,58 \mathrm{~b}$ & $0,54 \mathrm{~b}$ & $0,32 \mathrm{a}$ & 42,06 & 0,0238 \\
\hline \multirow{2}{*}{$\begin{array}{l}\text { HE } \\
(\%)\end{array}$} & $0-0,05$ & $24,68 \mathrm{~b}$ & $19,69 \mathrm{a}$ & $21,10 \mathrm{a}$ & 14,19 & 0,0063 \\
\hline & $0,05-0,10$ & $22,80 \mathrm{~b}$ & $18,29 \mathrm{a}$ & $20,71 \mathrm{ab}$ & 14,31 & 0,0127 \\
\hline \multirow{2}{*}{$\begin{array}{l}\text { EA } \\
(\%)\end{array}$} & $0-0,05$ & $86,61 \mathrm{~b}$ & $85,05 \mathrm{~b}$ & $63,05 \mathrm{a}$ & 15,35 & 0,0004 \\
\hline & $0,05-0,10$ & $84,67 \mathrm{~b}$ & $80,08 \mathrm{~b}$ & $51,20 \mathrm{a}$ & 17,96 & $<0,0001$ \\
\hline
\end{tabular}

La SD presentó una disminución de la EA del 24\% para los primeros $0,05 \mathrm{~m}$, y un 33\% para la siguiente profundidad respecto a la condición de alta calidad de suelo (Mon), con diferencias significativas respecto a Mon y SIL en las dos profundidades ( $<<0,0004 ; 0,0001)$. En la Región pampeana Norte, Ferreras et al. (2007), evaluaron suelos cultivados bajo siembra directa en comparación con el mismo tipo de suelo en su condición quasi-prístina, y encontraron una disminución en la estabilidad estructural y en el contenido de materia orgánica, destacando la estabilidad entre los indicadores de fertilidad física más sensibles. Sanzano et al. (2005), evaluaron en un Haplustol típico el efecto de 20 años de distintos sistemas de manejo en comparación con el suelo virgen y han encontrado que la estabilidad estructural se redujo a la mitad (sistema de siembra directa) y en algunos casos aún más (sistema de labranza convencional).

Por otra parte, Roldán et al. (2014), sostienen que los sistemas de labranza afectan a la agregación del suelo y su estabilidad, por acción directa y por alterar otros factores tales como la materia orgánica (MO). Comprobaron que suelos del orden Molisol de Balcarce sufrieron una disminución significativa de la estabilidad de agregados por el cambio en el uso, también afirman que la determinación de agregados estables podría ser un buen indicador de la salud de esos suelos. Para el tratamiento SIL no se encontraron diferencias significativas respecto al monte. El sistema silvopastoril produjo un impacto menor que el sistema agrícola, ya que, si bien provocó un aumento de la densidad aparente, y disminuyó la capacidad de retención de agua, el efecto sobre los agregados y su estabilidad fue menor, tomando valores medios entre 85 y $80 \%$, cercanos al Mon (85,64\%).

El efecto conjunto de una perturbación mínima y gran ingreso de residuos orgánicos frescos determina mejores condiciones para el funcionamiento del sistema silvopastoril (Silberman et al., 2015). Los SIL se caracterizan por ser altamente diversificados y autosuficientes, lo cual, asociado con un manejo agropecuario adecuado y acorde con las características del agroecosistema, favorecen procesos naturales e interacciones biológicas, que benefician procesos ecosistémicos fundamentales, tales como el ciclado de nutrientes, el secuestro de carbono, el mantenimiento de la estabilidad estructural, la fertilidad y consecuentemente su productividad (Vallejo, 2012). Silberman et al. (2015) evaluaron el efecto de un sistema silvopastoril con Panicum maximum cv Gatton panic (RBI), en Molisoles de la provincia de Santiago del Estero, sobre los contenidos orgánicos y la densidad aparente, y concluyeron que el RBI no afectó la calidad del suelo respecto al testigo (Monte nativo).

\section{CONCLUSIONES}

El desmonte y posterior uso agrícola bajo SD, provocó cambios desfavorables como densificación, disminución de la capacidad del suelo para retener agua para los cultivos y disminución de la estabilidad de los agregados, impactando negativamente sobre la calidad física.

En SIL, la incorporación de gramíneas resultó favorable y el sistema produjo un impacto menor en la calidad física, que el sistema agrícola.

La EA resultó un buen indicador de calidad, y de relativa facilidad de medición y bajo costo de determinación. El orden decreciente de calidad física del suelo en base al índice de estabilidad de agregados fue Mon $\geq$ Sil $>$ SD. 
Urinovsky Irigoyen, K.M.; et al. (2021) Indicadores de calidad física en suelos en suelos del Chaco semiárido...

\section{REFERENCIAS}

Angers, D.A. y Mehuys, G.R. (1988). Effects of cropping on macro-aggregation of a marine clay soil. Can. J. SoilSci. 68: 723.

Brejda, J.J., Moorman, T.B., Karlen, D.L. y Dao, T.H. (2000). Identification of regional soil quality factors and indicators: I. Central and Southern High Plains. Soil Sci. Soc. Am. J. 64: 2115-2124

Cabria, F., Calandroni, F. y Monterubbianesi, G. (2002). Tamaño y estabilidad de agregados y su relación con la conductividad hidráulica saturada en suelo bajo labranza convencional y praderas. Ciencia del Suelo 20 (2) 69-80.

Chenu, C. y Cosentino, D. (2011). Microbial regulation of soil structural dynamics, in The Architecture and Biology of Soils Life in Inner Space, K. Ritz and I. Young (Eds.). (Wallingford: CABI), 37-70.

Costa, O.Y.A., Raaijmakers J.M. y Kuramae E.E. (2018). Microbial Extracellular Polymeric Substances: Ecological Function and Impact on Soil Aggregation. Front. Microbiol. 9:1636.

Dalurzo, H.C. (2002). Agregado de residuos orgánicos en suelos ferralíticos. Efecto sobre variables que estiman sustentabilidad. Tesis de Magíster Scientiae. Área: Ciencia del Suelo. Escuela para Graduados Alberto Soriano UBA.

Dewis, J. y Freitas, F. (1970). Métodos físicos y químicos de análisis de suelos y aguas. Boletín sobre suelos $\mathrm{N}^{\mathrm{o}}$ 10. FAO. Roma. 36-57 pp.

Dexter, A.R. (1988). Advances in characterization of soil structure. SoilTillage Res. 11: 199-235.

Di Rienzo, J.A., Casanoves, F., Balzarini, M.G., Gonzalez, L., Tablada, M. y Robledo, C.W. (2017). Infostat, Versión 2017. Grupo InfoStat. FCA, UNC, Córdoba, Argentina.

Doran, J.W. y Parkin, T.B. (1994). Defining and assessing soil quality p. 3-21. In J.W. Doran et al (Ed.) Defining soil quality for a sustainable environment. SSSA Special Publ. 35. SSSA and ASA. Madison, WI.

Ferreras, L., Magra G., Besson, P., Kovalevski, E. y Garcia F. (2007). Indicadores de calidad física en suelos de la Región Pampeana Norte Argentina bajo siembra directa. Ciencia del Suelo 25(2): 159-172.

Forsythe, W. (1975). Física de Suelos. IICA. 212 p. San José. Costa Rica.

Golchin, J., Oades, M., Skemstad, J.O. y Clarke P. (1994). Soil structure and carbon cycling. Aust. J. Soil Res. 32:1043-1068.

González-Chávez, M.C.A., Gutiérrez-Castorena, M.C. y Wright, S. (2004). Hongos micorrízicos arbusculares en la agregación del suelo y su estabilidad. Terra Latinoamericana, vol. 22 (4): 507-514. Disponible en; http://www.redalyc.org/pdf/573/57311096014.pdf

Gutiérrez, N.C; Venialgo, C.; Gutiérrez J.R. (2002). Materia orgánica en sistemas de labranza y cultivos en la serie Tizón. Agrotecnia 9 (2002): 2-7.

Gregorich, E.G.; Carter, M.R.; Angers, D.A; Monreal C.M.; y B.H. Ellert. (1994). Towards a minimun data set to asses ssoil organic matter quality in agricultural soils. Can. J. Soil Sci. 74:367-385

Haynes, R.J. (2000). Labile organic matter as an indicator of organic matter quality in arable and pastoral soils in New Zeland. Soil Biol. Biochem. 32:211-219.

Kemper, W.D. y Rosenau, R.C. (1986). Aggregate stability and size distribution. En: A. Klute (ed.) Methods of soil analysis. Part 1. Physical and mineralogical methods. ASA and SSSA, Madison, WI. 425-441 pp.

Kooistra, M. y Tovey, N. (1991). Effects of compaction on soil microestructure. Chap 5 In: Soane and C van Ouwerkerk (Eds.) Soil Compaction in Crop Production Elsevier Science Conservación.

Kumar, R., Rawat, K. S., Singh, J., Singh, A. y Rai, A. (2013). Soil aggregation dynamics and carbon sequestration. J. Appl. Nat. Sci. 5: 250-267.

Ladd, J.N., Amato M., Li-kai, Z. y Schultz, J.E. (1994). Differential effects of rotation, plant residue and nitrogen fertilizer on microbial biomass and organic matter in an Australian Alfisol. Soil Biol. Biochem. 26:821-831.

Luters A. y Salazar, J.C. (1999). USDA. Guía para la evaluación de la calidad y salud del suelo. Departamento de Agricultura de los Estados Unidos de Norteamérica, Departamento de Agricultura, Servicio de Investigación Agrícola Servicio de Conservación de Recursos Naturales e Instituto de Calidad de Suelos, pp. $14-15,33,59-67$

Martino, D.L. (2017). Manejo de restricciones físicas del suelo en sistemas de siembra directa. Grupo de Riego, Agroclima, Ambiente y Agricultura Satelital (GRAS) del Instituto Nacional de Investigación Agropecuaria de Uruguay.

Montenegro González, H., Malagón Castro, D. y Guerrero, L. (1990). Propiedades Física de los suelos. Subdirección Agrológica. I.G.A.C. (Instituto Geográfico Agustín Codazzi). Bogotá, Colombia.

Montenegro, C., Gasparri, I., Manghi, E., Strada, M., Bono, J. y Parmuchi, M.G. (Diciembre, 2004). Informe Sobre Deforestación en Argentina. http://www.infobosques.com/descargas/biblioteca/151.pdf

Morello, J.H., Rodriguez, A.F. y Silva, M. (2009). Clasificación de Ambientes en Áreas Protegidas de las Ecorregiones del Chaco Húmedo y Chaco Seco. En: J.H., Morello, A.F. Rodríguez, (Eds.) El Chaco sin bosques: la Pampa o el desierto del futuro. $1^{\mathrm{a}}$ ed. Buenos Aires: Orientación Gráfica Editora. pp 53-91.

Moro, E.C., Venialgo, C.A., Gutierrez, N.C., Drganc, D., Asselborn, A. y Oleszczuk, J.D. (2004). Efecto de las labranzas y rotaciones sobre la compactación de suelos en distintos sistemas productivos de la provincia del Chaco-República Argentina. Agrotecnia 12 (2004): 1-8.

Oades, J. M. (1993). The role of biology in the formation, stabilization and degradation of soil structure. Geoderma 56: 377-400. 
Oades, J.M. (1984). Soil organic matter and structural stability: mechanisms and implications for management. Plant and Soil 76: 319-337.

Pla Sentís, I. y Ovalles, F. (1994). Efectos de los sistemas de labranza en la degradación y productividad de los suelos. FONAIAP. pp 3.

Porta Casanella, J.R., López-Acevedo, M. y Roquero de Laburu, C. (1999). Edafología. Para la agricultura y el medio ambiente. Ediciones Mundi-Prensa. 2da Edición.

Rojas, J.M. (2012). Indicadores de calidad de suelos desmontados y destinados a la producción agrícola en el área piloto de la Eco región Chaqueña. Maestría en Ciencias Agrarias, Orientación: Producción Sustentable. Facultad de Agronomía y Zootecnia. Universidad Nacional de Tucumán. 152 pp. Tesis de Maestría.

Rojas, J.M., Roldán, M.F. y Guevara, S.G. (2015). Influencia de rotaciones en la calidad del suelo en la zona central del Chaco y su relación con la producción de algodón. Ciencia del Suelo 33(2): 239-246

Roldán, M.A., Estuddert, G.A., Videla, C.D.C., San Martino, S. y Picone, L.I. (2014). Distribución de tamaño y estabilidad de agregados en Molisoles bajo labranzas contrastantes. Ciencia del Suelo 32 (2): 247-257

Rollán, A.C., Karlin, M.S. y Bachmeier, O.A. (2004). Siembra directa y densificación subsuperficial en molisoles del centro norte de Córdoba. XIX Congreso Argentino de la Ciencia del Suelo. Actas pp: 47.

Rossi, C.A. (2000). El Sistema Silvopastoril en la Región Chaqueña Árida y Semiárida Argentina Universidad Nacional de Lomas de Zamora. Argentina. https://www.produccion-animal.com.ar/ produccion_y_manejo_pasturas/manejo\%20silvopastoril/146-Reg_Chaque.pdf

Rosso, C.N. (2014). Una aproximación a la cuestión de la alimentación y la agricultura en el sudoeste de la provincia del Chaco: actores, políticas y problemáticas. Folia histórica del Nordeste (22): 65-87

Sansano, G.A., Corbella, R.D. y García J.R. (2005). Degradación física y química de un Haplustol típico bajo distintos sistemas de manejo de suelo. Ciencia del Suelo 23 (1):93-100.

Silberman, J.E., Anriquez, A.L., Domínguez, J., Kunts, C. y Albanesi, A.S. (2015). La cobertura arbórea en un sistema silvopastoril del Chaco y su contribución diferencial al suelo. Ciencia del Suelo 33(1): 19-29.

Six, J., Elliott E.T. y Paustian K. (2000). Soil macroaggregate turnover and microaggregate formation: a mechanism for $\mathrm{C}$ sequestration under no-tillage agriculture. Soil Biology and Biochemistry 32: 2099-2103.

Six, J., Bossuyt, H., Degryze S. y K Denef. (2004). A history of research on the link between (micro) aggregates, soil biota, and soil organic matter dynamics. SoilTillage Res. 79: 7-31.

Toledo, D.M. (2014). Calidad de Suelo en Agro-ecosistemas de Misiones: Desarrollo y Validación de Calidad. $\mathrm{Su}$ Aplicación en la Evaluación de los Cambios en el Uso de las Tierras. Tesis de Doctorado en el área de Recursos Naturales. Facultad de Ciencias Agrarias. Universidad Nacional del Nordeste.

USDA. (1996). Soil Quality Indicators: Aggregate Stability. Soil Quality Information Sheet. April 1996. USDA Natural Resources Conservation Service.

USDA. (1999). Guía para la Evaluación de la Calidad y Salud del Suelo. Departamento de Agricultura Servicio de Investigación Agrícola Servicio de Conservación de Recursos Naturales Instituto de Calidad de Suelos. Agosto, 1999.

USDA. (2011). Aggregate stability. NRCS East National Technology Support Center, NRCS National Soil Survey Center, ARS National Laboratory for Agriculture and the Environment, Department of Natural Resources and Environmental Sciences, University of Illinois at Urbana-Champaign. http:// soilquality.org/indicators/aggregate_stability.html.

Utomo, W.H. y Dexter, A.R. (1982). Changes in soil aggregate water stability induced by wetting and drying cycles in non-saturated soil. Soil Sci. 33: 623- 637.

Vallejo, V. (2012). Efecto del establecimiento de sistemas silvopastoriles sobre la comunidad microbiana edáfica (total y de bacterias oxidadoras de amonio) en la Reserva Natural: El Hatico-Valle (Tesis doctoral). Bogotá: Pontificia Universidad Javeriana. 239 p.

Venialgo, C.A., Gutiérrez, N.C. y José, R. (2004). Variables edáficas en diferentes sistemas de uso del suelo en el sudoeste chaqueño. Jornadas de Comunicaciones Científicas y Tecnológicas 2004. Resumen expandido A-074. Universidad Nacional del Nordeste. https://studylib.es/doc/6679900/variables-edaficas-endiferentes-sistemas-de-uso-del-suelo. .

Vigglizzo, E.F. y Jobbágy, E. (2010). Expansión de la Frontera Agropecuaria en Argentina y su Impacto Ecológico - Ambiental. Buenos Aires. Ediciones INTA. 106 pp. https://inta.gob.ar/sites/default/files/scripttmp-expansin_frontera_agropecuaria_2010.pdf 